\title{
Calculating fifth-order Raman signals for various molecular liquids by equilibrium and nonequilibrium hybrid molecular dynamics simulation algorithms
}

\author{
$\operatorname{AUTHOR}(\mathrm{S}):$ \\ Hasegawa, T; Tanimura, $Y$
}

\section{CITATION:}

Hasegawa, $T$... [et al]. Calculating fifth-order Raman signals for various molecular liquids by equilibrium and nonequilibrium hybrid molecular dynamics simulation algorithms. JOURNAL OF CHEMICAL PHYSICS 2006, 125(7): 074512.

\section{ISSUE DATE:}

2006-08-21

URL:

http://hdl.handle.net/2433/49988

\section{RIGHT:}

Copyright 2006 American Institute of Physics. This article may be downloaded for personal use only. Any other use requires prior permission of the author and the American Institute of Physics. 


\title{
Calculating fifth-order Raman signals for various molecular liquids by equilibrium and nonequilibrium hybrid molecular dynamics simulation algorithms
}

\author{
Taisuke Hasegawa ${ }^{\text {a) }}$ and Yoshitaka Tanimura ${ }^{\text {b) }}$ \\ Department of Chemistry, Graduate School of Science, Kyoto University, Sakyo, Kyoto 606-8502, Japan
}

(Received 27 March 2006; accepted 2 June 2006; published online 21 August 2006)

\begin{abstract}
The fifth-order two-dimensional (2D) Raman signals have been calculated from the equilibrium and nonequilibrium (finite field) molecular dynamics simulations. The equilibrium method evaluates response functions with equilibrium trajectories, while the nonequilibrium method calculates a molecular polarizability from nonequilibrium trajectories for different pulse configurations and sequences. In this paper, we introduce an efficient algorithm which hybridizes the existing two methods to avoid the time-consuming calculations of the stability matrices which are inherent in the equilibrium method. Using nonequilibrium trajectories for a single laser excitation, we are able to dramatically simplify the sampling process. With this approach, the 2D Raman signals for liquid xenon, carbon disulfide, water, acetonitrile, and formamide are calculated and discussed. Intensities of 2D Raman signals are also estimated and the peak strength of formamide is found to be only five times smaller than that of carbon disulfide. (C) 2006 American Institute of Physics.
\end{abstract}

[DOI: $10.1063 / 1.2217947]$

\section{INTRODUCTION}

Understanding the dynamics of molecules in condensed phases has been the central issues in chemistry, physics, and biology. Since intra- and intermolecular vibrational motions are extremely fast, femtosecond laser spectroscopy is suitable to investigate roles of the vibrational motions. Much physical insight can be gained by formulating nonlinear spectroscopy in terms of nonlinear response functions which are defined by the correlation functions of molecular dipole moment or polarizability. ${ }^{1}$ In complex media such as molecular liquids and biological systems, it is shown that multidimensional vibrational spectroscopy is a valuable tool because of its sensitivity of multitime response functions of optical observables. The fifth-order two-dimensional (2D) $\operatorname{Raman}^{2-5}$ and third-order 2D IR spectroscopies ${ }^{6-11}$ are such examples. In linear spectroscopy which is defined by a twobody response function, the main contributions of a signal arises from harmonic vibrational motions and anharmonic effects are merely the small correction. On the contrary, in higher-order vibrational spectroscopy, the anharmonicity of potential $^{12-16}$ and the anharmonic mode coupling ${ }^{17-21}$ as well as the dephasing process ${ }^{22-27}$ are essential to characterize a signal. Therefore, one may consider that linear vibrational spectroscopies probe the character of harmonic vibrational motions, whereas higher-order vibrational spectroscopies detect anharmonic dynamics. ${ }^{27}$

To investigate the nature of intermolecular interactions, 2D Raman spectroscopy which was originally proposed to distinguish between homogeneous and inhomogeneous broadenings of liquid dynamics is advantageous, because

\footnotetext{
${ }^{a)}$ Electronic mail: hasegawa@kuchem.kyoto-u.ac.jp

${ }^{b)}$ Electronic mail: tanimura@kuchem.kyoto-u.ac.jp
}

Raman pulses can create instantaneous vibrational excitations on a molecular system and their coherence can be detected by spectroscopic means. This experiment uses two pairs of Raman excitation pulses separated by period $t_{1}$, followed by a probe pulse to generate the polarizability after another period $t_{2}$, and therefore has two time variables. ${ }^{28}$ The signals corresponding to various fifth-order Raman polarizability tensor elements have been measured for the intermolecular vibrational modes of liquids $\mathrm{CS}_{2}$ by utilizing phase matching conditions and pulse geometry. ${ }^{3-5}$ Although molecular dynamics (MD) simulation techniques have also been developed and their results have been compared with the experimental results, the position of the nodal plane predicted by the equilibrium MD simulations has not been resolved. ${ }^{29-32}$

The fifth-order Raman experiment is probably one of the most difficult experiments in the spectroscopy and the signals obtained are only for $\mathrm{CS}_{2}$ liquids that exhibit a strong Raman polarizability. While experimentalists have made efforts to obtain signals, theorists have been intrigued by this subject in spite of those difficulties, since 2D Raman signals can reveal an interesting nonlinear dynamics of the system which arises from the stability matrix involved in the nonlinear response function. Thus, it has been shown that an appropriate representation of the 2D Raman measurements provides an interpretable depiction of the structural and dynamical properties in relation to the inhomogeneous distribution, ${ }^{2}$ the anharmonicity, ${ }^{12-16}$ mode coupling mechanisms, ${ }^{17-21}$ inter- and intramolecular vibrational motions, ${ }^{32-36}$ relaxation mechanisms, ${ }^{22-27,37,38}$ and wave packet dynamics. ${ }^{16,27}$ The nature of the stability matrix ${ }^{39,40}$ and the phenomenological simulation method to calculate the fifth-order signals such as the Langevin treatment have been investigated. ${ }^{41-44}$ Since the experimental results are limited 
to the case of $\mathrm{CS}_{2}$, the theoretical models and the phenomenological simulation methods mentioned above have to be justified by comparing the signal accurately calculated from the MD simulations.

Nevertheless the signals calculated from full MD simulations are limited to the case of $\mathrm{Xe}^{33,45,46} \mathrm{CS}_{2}{ }^{29-31,47}$ $\mathrm{H}_{2} \mathrm{O},{ }^{31}$ and atomic liquids and solids with soft core potential. $^{48,49}$ This is because calculating 2D Raman response function is time consuming due to the sensitivity of the simulation conditions and numerical errors.

There are two methods to simulate the two-dimensional Raman signals from the full MD simulations: the equilibrium and nonequilibrium (finite field) approaches. The equilibrium MD approach computes a nonlinear optical response function expressed in the multiple Poisson brackets of the equilibrium molecular trajectories. The difficulty of this method is on the evaluation of the stability matrix involved in the response function. Since the stability matrix is very sensitive to the difference of trajectories, one has to take a large assemble of the stability matrix elements. Therefore large storage space and powerful CPUs are required to calculate the stability matrix. The nonequilibrium MD (NEMD) approach performs the fifth-order Raman experiment on the computer. Raman polarizability is directly calculated from nonequilibrium MD trajectories under a pair of external laser pulses with different time sequences. The method does not require the calculations of the stability matrix; however, to separate the fifth-order Raman signal from the polarizability, we have to subtract lower-order contributions separately calculated from different nonequilibrium trajectories with fewer excitation pulses. Since we have to generate many trajectories to change the time sequences and to separate the lower-order contributions, this method requires strong CPU power. The calculations of signals from both approaches are also examined using the different expressions of the nonlinear response function where the stability matrix is eliminated, but the results are still limited to a simple liquid with simple laser pulse sequences. ${ }^{50}$

Because the computer resources have limited the calculations of the signals from both approaches, we have developed an efficient algorithm which hybridizes the existing equilibrium and nonequilibrium approaches. In this hybrid method, the fifth-order Raman signal is calculated from a single Poisson bracket of the nonequilibrium trajectories with a single Raman excitation. The second Raman excitation part of the fifth-order response function is taken into account by the nonequilibrium trajectories, while the first excitation and calculations of Raman polarizability are taken into account by the evaluations of the Poisson bracket. This hybrid method enables us to avoid the time-consuming calculations of the stability matrices. We then calculate the fifth-order Raman signals for $\mathrm{Xe}, \mathrm{CS}_{2}$, and water to compare with previously obtained results to check the accuracy and efficiency, and for acetonitrile and formamide to explore a nature of molecular liquids.

In Sec. II, we explain our simulation method for the fifth-order Raman response function. In Sec. III, we show the results of simulations for various molecular liquids. We then discuss the simulation results in Sec. IV. Section V is devoted to concluding remarks.

\section{EQUILIBRIUM AND NONEQUILIBRIUM HYBRID ALGORITHMS}

We consider a liquid system expressed by the Hamiltonian $H_{0}(\mathbf{p}, \mathbf{q})$, where $\mathbf{p}$ and $\mathbf{q}$ are the molecular momenta and coordinates, respectively. In the fifth-order off-resonant Raman experiment, the system interacts with two pairs of off-resonant laser pulses. In order to illustrate our method, we divide the total Hamiltonian $H(t)$ as

$$
H(t)=H_{c d}^{\prime}(t)+V(t),
$$

where

$$
\begin{aligned}
& H_{c d}^{\prime}(t)=H_{0}(\mathbf{p}, \mathbf{q})-\frac{1}{2} E_{c}(t) \Pi_{c d}(\mathbf{q}) E_{d}(t), \\
& V(t)=-\frac{1}{2} E_{a}(t) \Pi_{a b}(\mathbf{q}) E_{b}(t),
\end{aligned}
$$

in which $\Pi_{a b}$ and $\Pi_{c d}$ are the $a b$ and $c d$ tensor elements of the polarizability, respectively. The distribution function of molecules $W(\mathbf{p}, \mathbf{q} ; t)$ then follows the Liouville equation,

$$
\frac{\partial}{\partial t} W(\mathbf{p}, \mathbf{q} ; t)=-\left[\hat{L}_{c d}^{\prime}(t)+\hat{L}_{V}(t)\right] W(\mathbf{p}, \mathbf{q} ; t) .
$$

The Liouvillian for $H_{c d}^{\prime}(t)$ and $V(t)$ are expressed as

$$
\hat{L}_{c d}^{\prime}(t) W(\mathbf{p}, \mathbf{q} ; t) \equiv\left\{H_{c d}^{\prime}(t), W(\mathbf{p}, \mathbf{q} ; t)\right\}_{\mathrm{PB}},
$$

and

$$
\hat{L}_{V}(t) W(\mathbf{p}, \mathbf{q} ; t) \equiv\{V(t), W(\mathbf{p}, \mathbf{q} ; t)\}_{\mathrm{PB}},
$$

respectively, where \{\}$_{\mathrm{PB}}$ is the Poisson bracket defined by

$$
\{A, B\}_{\mathrm{PB}} \equiv \frac{\partial A}{\partial q} \frac{\partial B}{\partial p}-\frac{\partial A}{\partial p} \frac{\partial B}{\partial q},
$$

for any functions $A$ and $B$. We introduce the time evolution operator of the Liouvillian $L_{c d}^{\prime}(t)$ given by

$$
\hat{U}_{c d}^{\prime}\left(t_{f}-t_{i}\right)=\exp _{+}\left[-\int_{t_{i}}^{t_{f}} d \tau \hat{L}_{c d}^{\prime}(\tau)\right]
$$

where $\exp _{+}[]$is positive time ordered exponential. The observable of Raman measurement is a Raman polarizability tensor given by

$$
\Pi_{e f}(t)=\operatorname{Tr}\left\{\Pi_{e f}(\mathbf{q}) W(\mathbf{p}, \mathbf{q} ; t)\right\},
$$

where $W(\mathbf{p}, \mathbf{q} ; t)$ follows Eq. (4). Expanding the polarizability in Eq. (9) in powers of only $L_{V}(t)$, we have

$$
\Pi_{e f}(t)=S_{e f}(t)+\frac{1}{2} \int_{0}^{\infty} d \tau E_{a}(t-\tau) E_{b}(t-\tau) R_{e f ; a b}(\tau)+\cdots,
$$

where

$$
S_{e f}(t) \equiv\left\langle\Pi_{e f}(\mathbf{q}) \hat{U}_{c d}^{\prime}(t) W^{\mathrm{eq}}(\mathbf{p}, \mathbf{q})\right\rangle,
$$

and 


$$
R_{e f ; a b}(\tau) \equiv \theta(\tau)\left\langle\Pi_{e f}(\mathbf{q}) \hat{U}_{c d}^{\prime}(\tau)\left\{\Pi_{a b}(\mathbf{q}), W^{\mathrm{eq}}(\mathbf{p}, \mathbf{q})\right\}_{\mathrm{PB}}\right\rangle .
$$

Here, $W^{\mathrm{eq}}(\mathbf{p}, \mathbf{q})$ is the equilibrium distribution function and $\theta(\tau)$ is the Heaviside step function. Equation (12) is the expression that we are going to use. The time correlation function in Eq. (12) is defined by the nonequilibrium trajectories. Suppose if the second Raman excitation in $\hat{U}_{c d}^{\prime}(t)$ is treated perturbatively, $R_{e f ; a b}(\tau)$ is further expanded as

$$
\begin{aligned}
R_{e f: a b}(\tau)= & R_{e f: a b}^{(3)}(\tau)+\frac{1}{2} \int_{0}^{\infty} d \tau^{\prime} E_{c}\left(t-\tau^{\prime}\right) E_{d}\left(t-\tau^{\prime}\right) \\
& \times R_{e f ; c d: a b}^{(5)}\left(\tau, \tau^{\prime}\right)
\end{aligned}
$$

where

$$
R_{e f: a b}^{(3)}(\tau) \equiv \theta(\tau)\left\langle\Pi_{e f}(\mathbf{q}) \hat{U}_{0}(\tau)\left\{\Pi_{a b}(\mathbf{q}), W^{\mathrm{eq}}(\mathbf{p}, \mathbf{q})\right\}_{\mathrm{PB}}\right\rangle,
$$

and

$$
\begin{aligned}
R_{e f ; c d: a b}^{(5)}\left(\tau, \tau^{\prime}\right)= & \theta(\tau) \theta\left(\tau^{\prime}\right)\left\langle\Pi _ { e f } ( \mathbf { q } ) \hat { U } _ { 0 } ( \tau ) \left\{\Pi_{c d}(\mathbf{q}), \hat{U}_{0}\left(\tau^{\prime}\right)\right.\right. \\
& \left.\left.\times\left\{\Pi_{a b}(\mathbf{q}), W^{\mathrm{eq}}(\mathbf{p}, \mathbf{q})\right\}_{\mathrm{PB}}\right\}_{\mathrm{PB}}\right\rangle .
\end{aligned}
$$

$\hat{U}_{0}(t)$ is the time evolution operator without external fields, i.e., $\hat{U}_{c d}^{\prime}(t)$ in Eq. (8) with $E_{c}(t)=E_{d}(t)=0$. The expressions in Eqs. (13)-(15) indicate that we can calculate the fifth-order Raman signal from Eq. (12) by evaluating the Poisson bracket and by subtracting the third-order contribution $R_{e f: a b}^{(3)}(\tau)$ if we obtain the nonequilibrium trajectories under the external fields $E_{c}\left(t-\tau^{\prime}\right) E_{d}\left(t-\tau^{\prime}\right)=E_{c} E_{d} \delta\left(t-\tau^{\prime}\right)$. Since Eq. (12) does not contain the stability matrix and the nonequilibrium trajectories used in this method contain only one external force, the requirement of CPU power is much lower than in the equilibrium and NEMD approaches. Hereafter, we call this method, the equilibrium and nonequilibrium hybrid approach.

Since our method partially relies on the NEMD method, the present fifth-order signal may also be contaminated by a seventh-order signal in a case of the strong electric fields. Thus, we apply the inverse force method developed for the NEMD approach. ${ }^{47}$ This method utilizes the expressions of the fifth-order polarizability defined by

$$
\begin{aligned}
\Pi_{e f ; c d ; a b}^{(5)}(t) \propto & \Pi_{e f ; c d ; a b}(t)-\Pi_{e f ; \bar{c} d ; a b}(t)-\Pi_{e f ; c d ; \bar{a} b}(t) \\
& +\Pi_{e f ; \bar{c} ; \bar{a} b}(t),
\end{aligned}
$$

where $\bar{a}$ and $\bar{c}$ denote the inverted polarization directions of the radiation fields. To use the inverted force method, we first define

$$
\Delta R_{e f ; a b}(\tau) \equiv R_{e f: a b}(\tau)-\bar{R}_{e f: a b}(\tau),
$$

where

$$
\bar{R}_{e f ; a b}(\tau) \equiv \theta(\tau)\left\langle\Pi_{e f}(\mathbf{q}) \hat{U}_{\bar{c} d}^{\prime}(\tau)\left\{\Pi_{a b}(\mathbf{q}), W^{\mathrm{eq}}(\mathbf{p}, \mathbf{q})\right\}_{\mathrm{PB}}\right\rangle .
$$

Using the relation, $\quad\left\{\Pi(\mathbf{q}), W^{\mathrm{eq}}(\mathbf{p}, \mathbf{q})\right\}_{\mathrm{PB}}$ $=W^{\text {eq }}(\mathbf{p}, \mathbf{q}) \dot{\Pi}(\mathbf{q}) / k_{B} T$, we have

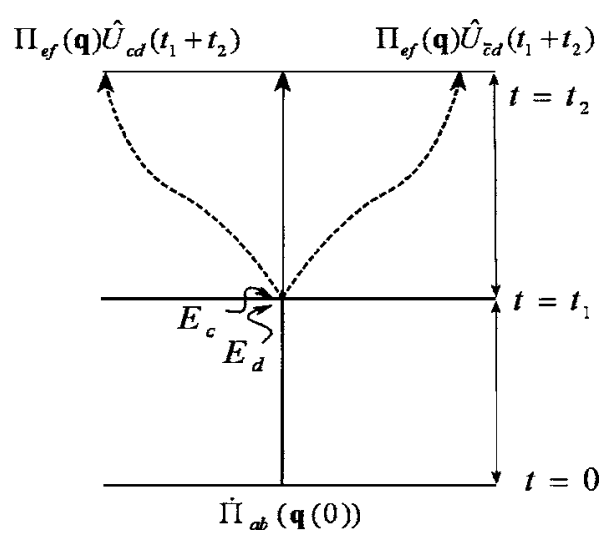

FIG. 1. The sampling procedure in the hybrid method. The solid line denotes the equilibrium trajectories and the doted lines denote the trajectories perturbed by laser pulses.

$$
\Delta R_{e f ; a b}(\tau)=\frac{1}{k_{B} T} \theta(\tau)\left\langle\left(\Pi_{e f}(\mathbf{q}) \hat{U}_{c d}^{\prime}(\tau)-\Pi_{e f}(\mathbf{q}) \hat{U}_{\bar{c} d}^{\prime}(\tau)\right)\right.
$$

$$
\left.\times \dot{\Pi}_{a b}(\mathbf{q}) W^{\mathrm{eq}}(\mathbf{p}, \mathbf{q})\right\rangle .
$$

The merit of the inverse force method is discussed in Appendix.

If we assume $E_{a}(t) E_{b}(t)=E_{a} E_{b} \delta(t)$ and $E_{c}(t) E_{d}(t)$ $=E_{c} E_{d} \delta\left(t-t_{1}\right)$, we have the efcdab tensor element of the fifth-order response function as

$$
\begin{aligned}
R_{e f ; c d ; a b}^{(5)}\left(t_{2}, t_{1}\right) \equiv & \frac{1}{k_{B} T E_{c} E_{d}}\left\langle\left(\Pi_{e f}(\mathbf{q}) \hat{U}_{c d}^{\prime}\left(t_{2}+t_{1}\right)\right.\right. \\
& \left.\left.-\Pi_{e f}(\mathbf{q}) \hat{U}_{\bar{c} d}^{\prime}\left(t_{2}+t_{1}\right)\right) \dot{\Pi}_{a b}(\mathbf{q}) W^{\mathrm{eq}}(\mathbf{p}, \mathbf{q})\right\rangle,
\end{aligned}
$$

where $t_{2}=t-t_{1}$. This expression allows us to calculate the fifth-order response function from one equilibrium MD trajectory and two NEMD trajectories perturbed by a Raman excitation pulse.

\section{COMPUTATIONAL DETAILS AND RESULTS}

To illustrate our method, we have calculated the fifthorder Raman signals for several molecular liquids. We first evaluate the time derivative of the polarizability $\dot{\Pi}_{a b}(\mathbf{q}(0))$ using the equilibrium MD simulation trajectories. To calculate the polarizability $\Pi_{e f} \hat{U}_{c d}\left(\mathbf{q}\left(t_{1}+t_{2}\right)\right)$, we perform NEMD simulations with laser pulses, $E_{c} E_{d} \delta\left(t-t_{1}\right)$, started from the initial configurations sampled from equilibrium MD trajectories. In the same way, we calculate $\Pi_{e f} \hat{U}_{\bar{c} d}\left(\mathbf{q}\left(t_{1}+t_{2}\right)\right)$ for the opposite direction of laser polarization $c$ to apply the inverse force method. Finally, the fifth-order signal is calculated from Eq. (20). This procedure is shown schematically in Fig. 1.

We have used the constant volume and constant energy calculation for equilibrium MD simulations. In the NEMD calculations, we have used the Hamiltonian represented by Eq. (2); therefore, the total energies of the system were not conserved. The MD simulations were carried out with the periodic boundary condition in a cubic box. The total polarizability was calculated for $\mathrm{CS}_{2}$ and the other molecules by the full-order dipole-induced dipole and first-order dipoleinduced dipole models, respectively. ${ }^{51}$ The polarizability 

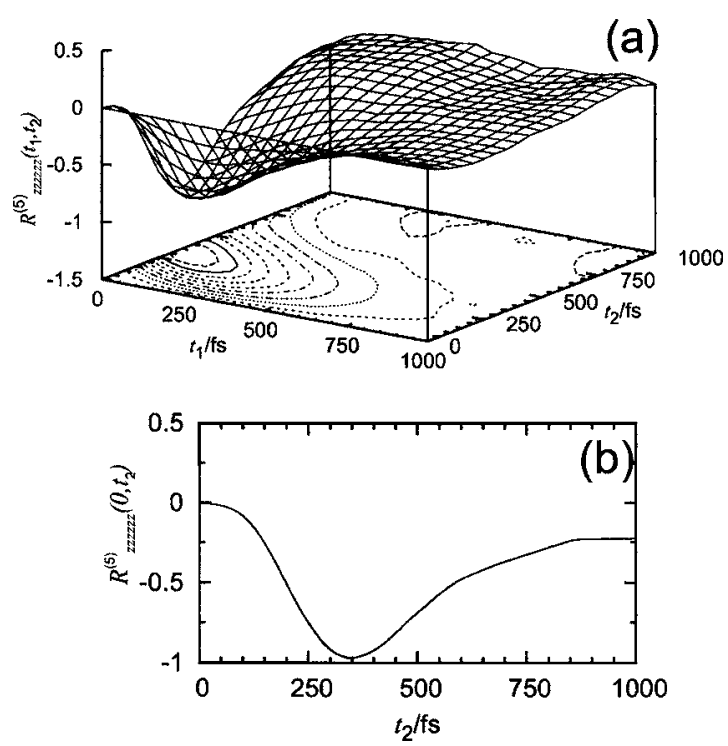

FIG. 2. (a) The $z z z z z z$ tensor element of the fifth-order response function of Xe. The intensity of the signal is normalized by the peak intensity of the signal. (b) The slice of the $z z z z z z$ tensor element along the $t_{2}$ axis.

from intramolecular motions were neglected, so that only the intermolecular motions contribute to the response functions. Interactions of potential energy and polarizability were cut off smoothly at the half length of the simulation boxes with a switching function. The long range Coulomb interactions were calculated by the Ewald sum. Note that by changing the number of molecules we checked the size dependence of the simulations without the Ewald sum. Since the convergence of the fifth-order response function depends on the strength of applied laser fields, we have repeated the NEMD calculations for appropriate laser strength until the signals converged (see Appendix). In the following examples, except for the Xe case, the molecules have been treated as rigid bodies and the equation of motion has been solved by the symplectic integrator. ${ }^{52}$ We summarize the computational details and results for each of the molecular liquids as follows.

\section{A. Liquid Xe}

To carry out MD simulation, we employed the same potential and polarizability for liquid Xe that were used by Ma and Stratt. ${ }^{45}$ We changed the number of molecules and found that there was no significant difference between the signal with 64 molecules and that with 216 molecules; we used 108 molecules. The equations of motion were solved by the velocity-Verlet integrator with the time step of $5 \mathrm{fs}$. The box lengths were determined for reduced density to be 0.74 . The MD simulations were performed at the average temperature of $220 \mathrm{~K}$. The NEMD calculation was carried out with laser fields of $5.0 \mathrm{~V} / \AA$. The fifth-order response function was obtained by averaging over $10^{7}$ initial configurations.

The $z z z z z z$ tensor element of the fifth-order response function of liquid $\mathrm{Xe}$ is shown in Fig. 2(a). It has a large signal along $t_{2}$ axis and the peak position is located around $\left(t_{1}, t_{2}\right) \approx(50,330)$ fs. Small noises in the region $t_{2}>500 \mathrm{fs}$ may be attributed to the poor convergence of the response function. The slice of the signals along the $t_{2}$ axis is shown in Fig. 2(b). The signal is peaked at $t_{2} \approx 350 \mathrm{fs}$ and then decays
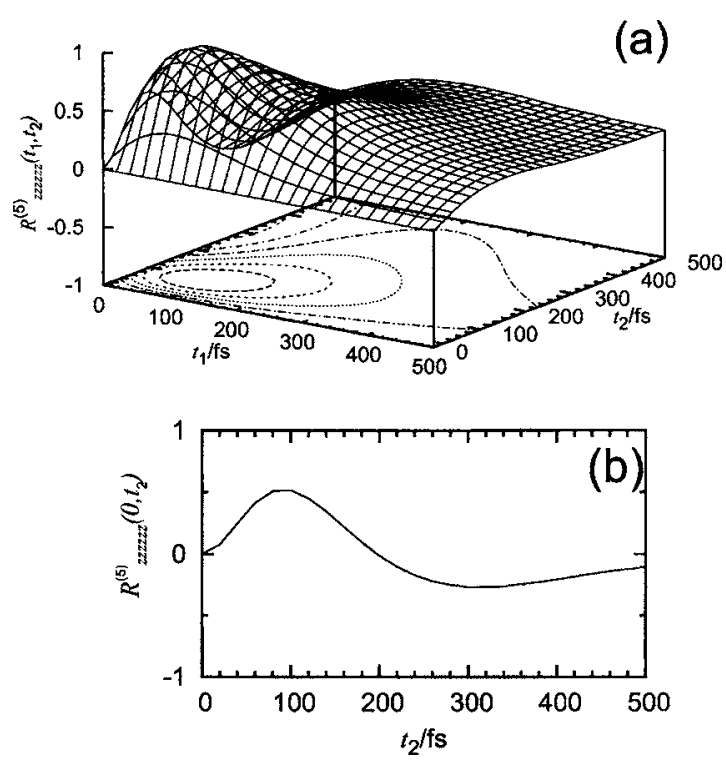

FIG. 3. The $z z z z z z$ tensor element of the fifth-order response function of $\mathrm{CS}_{2}$. The intensity of the signal is normalized by the peak intensity of the signal. (b) The slice of the $z z z z z z$ tensor element along the $t_{2}$ axis.

slowly. Here, we plot the response function $R_{z z z z z z}^{(5)}\left(t_{1}, t_{2}\right)$, but if we calculate the intensity $I_{z z z z z z}^{(5)}\left(t_{1}, t_{2}\right)=\left|R_{z z z z z z}^{(5 z z z z z}\left(t_{1}, t_{2}\right)\right|^{2}$, the present result agrees with Ma and Stratt's results.

\section{B. $\mathrm{CS}_{2}$}

We used the polarizability introduced by Bogaard et al. for $\mathrm{CS}_{2}$ (Ref. 53) and intermolecular potential by Moore and Keys. ${ }^{54}$ We changed the number of molecules and found that there was no significant difference between the signal with 32 molecules and that with 216 molecules; we used 108 molecules in this calculation. The box lengths were determined for density to be $1.27 \mathrm{~g} / \mathrm{cm}^{3}$. The calculation was carried out by the symplectic integrator with the time step of $2 \mathrm{fs}$. The MD simulations were performed at the average temperature of $270 \mathrm{~K}$. The intensity of the applied laser fields was $5.0 \mathrm{~V} / \AA$ in the NEMD calculation. We checked the convergence of the signal and found that the averaging over $1.5 \times 10^{6}$ initial configurations were enough to obtain the reliable signal.

The $z z z z z z$ tensor element of the fifth-order signal of $\mathrm{CS}_{2}$ is shown in Fig. 3(a). The signal is peaked around $\left(t_{1}, t_{2}\right)$ $\approx(90,90)$ fs. There is a node around $t_{2}$ axis and a negative signal at $t_{2}>200 \mathrm{fs}$, which shows very good agreement with the result of the other MD calculations which use the same potential. $^{31,32}$ This comparison helps confirm the accuracy of the new hybrid methods.

\section{Water}

To simulate water, the TIP4P potential ${ }^{55}$ and Huiszoon's polarizability ${ }^{56}$ were used. We calculated the signals with 216, 108, and 64 waters and found that 108 waters were large enough to have reliable 2D Raman signals; we used 108 waters. Equations of motion were solved with the time step of $1 \mathrm{fs}$. The box lengths were determined for density to be $1.0 \mathrm{~g} / \mathrm{cm}^{3}$. The MD simulations were performed at the average temperature of $300 \mathrm{~K}$. 

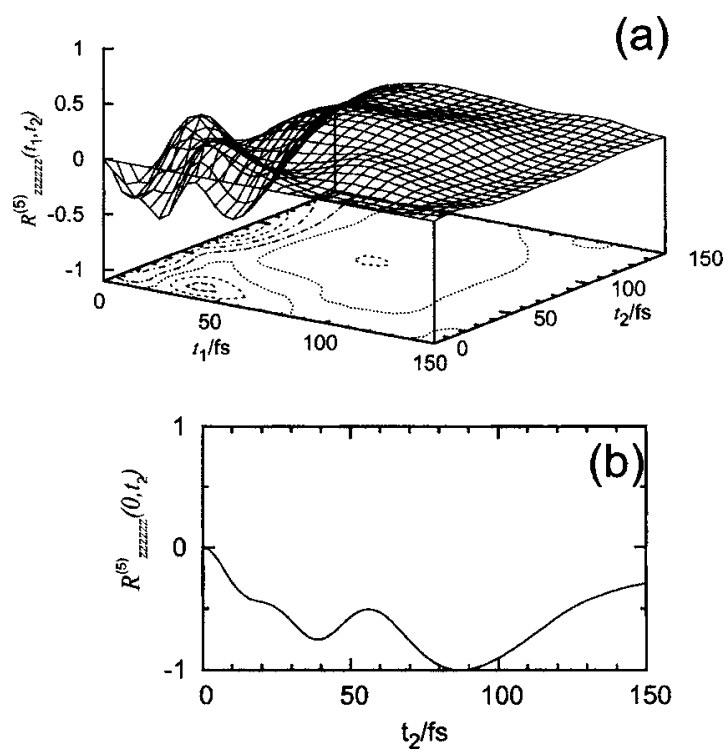

FIG. 4. (a) The $z z z z z z$ tensor element of the fifth-order response function of water. The intensity of the signal is normalized by the peak intensity of the signal. (b) The slice of the $z z z z z z$ tensor element along the $t_{2}$ axis.

The intensity of the applied laser fields was $15 \mathrm{~V} / \AA$ in the NEMD calculation. The fifth-order response function was obtained by averaging over $4 \times 10^{6}$ initial configurations.

The $z z z z z z$ tensor element of the fifth-order response function of water is given in Fig. 4(a). We can see a positive peak around $\left(t_{1}, t_{2}\right) \approx(35,15)$ fs and a strong ridge which consists of three negative peaks. Clear peaks around $t_{2} \approx 40$ and $90 \mathrm{fs}$, and a shoulder around $t_{2} \approx 20 \mathrm{fs}$ are observed in Fig. 4(b). This result is in good agreement with the result by Saito and Ohmine, where the fifth-order response function is calculated by equilibrium MD simulation. ${ }^{31}$

\section{Formamide}

The modified $T$-model potential ${ }^{57,58}$ was used in the calculation of formamide. We used the polarizability in Ref. 59 and assumed the molecular polarizability of formamide resides in the center of mass of the molecule. We calculated the signals with 216, 108, and 64 formamide molecules and found that 108 molecules were large enough to have reliable 2D Raman signals; we used 108 molecules. To solve the equation of motion, the time step was chosen to be $2 \mathrm{fs}$. The box lengths were determined for density to be $1.12 \mathrm{~g} / \mathrm{cm}^{3}$ and the intensity of the applied laser fields was $5 \mathrm{~V} / \AA$ in the NEMD calculation. The MD simulations were performed at the average temperature of $300 \mathrm{~K}$. The fifth-order response function was obtained by averaging over $2 \times 10^{6}$ initial configurations.

The $z z z z z z$ tensor element of the fifth-order response function of formamide is given in Fig. 5(a). We have observed a positive peak around $\left(t_{1}, t_{2}\right) \approx(120,140)$ fs and a negative peak around $\left(t_{1}, t_{2}\right) \approx(20,70)$ fs.

\section{E. Acetonitrile}

We used the intermolecular potential function by Ref. 60 and molecular polarizability by Ref. 59 . We calculated the signals with 216, 108, and 64 acetonitrile molecules and
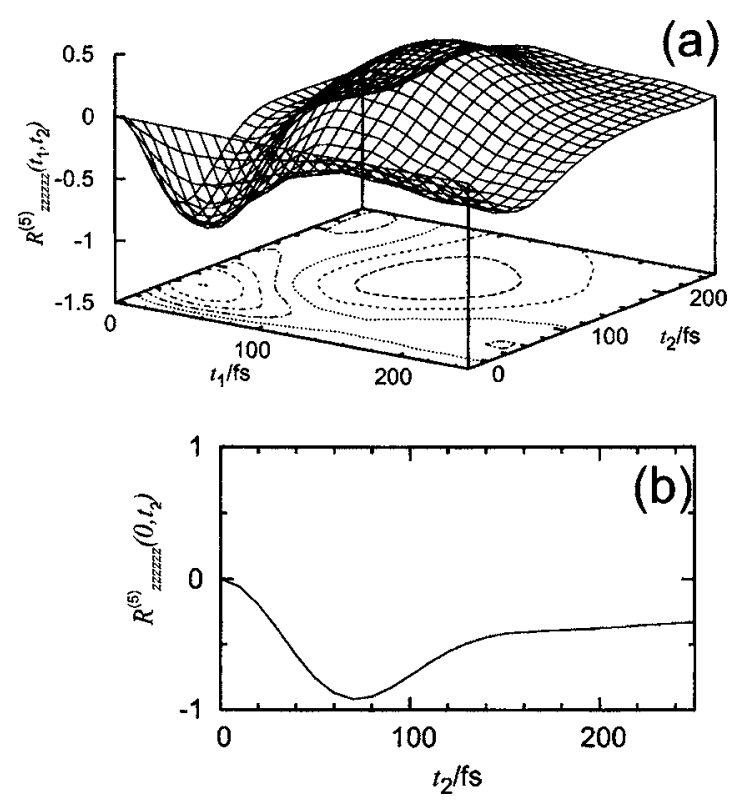

FIG. 5. (a) The $z z z z z z$ tensor element of the fifth-order response function of formamide. The intensity of the signal is normalized by the peak intensity of the signal. (b) The slice of the $z z z z z z$ tensor element along the $t_{2}$ axis.

found that 64 molecules were large enough to have reliable 2D Raman signals; we calculated the signal with 108 molecules to compare with the signals from the other molecules.

Equation of motion was solved with the time step of 2 fs. The box lengths were determined for density to be $0.777 \mathrm{~g} / \mathrm{cm}^{3}$ and the intensity of the applied laser fields was $5 \mathrm{~V} / \AA$ in the NEMD calculation. The MD simulations were performed at the average temperature of $300 \mathrm{~K}$. The fifthorder response function was obtained by averaging over 2 $\times 10^{6}$ initial configurations.

The $z z z z z z$ tensor element of the fifth-order response function of acetonitrile is given in Fig. 6(a). While acetonitrile molecule has a dipole moment unlike $\mathrm{CS}_{2}$, the profile of the fifth-order signal of this molecule is similar to that of $\mathrm{CS}_{2}$. In Fig. 6(a), we observe a peak around $\left(t_{1}, t_{2}\right)$ $\approx(80,80)$ fs. In Fig. 6(b), a negative signal at $t_{2}>150 \mathrm{fs}$ is observed.

\section{DISCUSSION}

To explore the possibility of detecting signals experimentally, we have evaluated the relative intensities of the fifth and third-order Raman signals of liquids. The thirdorder response functions were calculated by the equilibrium MD simulation and the fifth-order response functions were calculated by the hybrid method. The results are listed in Table I. It is shown that the fifth-order signal of formamide is much stronger than that of water, liquid $\mathrm{Xe}$, or acetonitrile and is only five times smaller than that of $\mathrm{CS}_{2}$. This is because $\mathrm{CS}_{2}$ and formamide have the strong anisotropy of the polarizability. Water has much smaller anisotropy and shows much smaller signals than $\mathrm{CS}_{2}$ and formamide. Moreover, $\mathrm{Xe}$ which has only the isotropic polarizability shows the weakest fifth-order signal. 

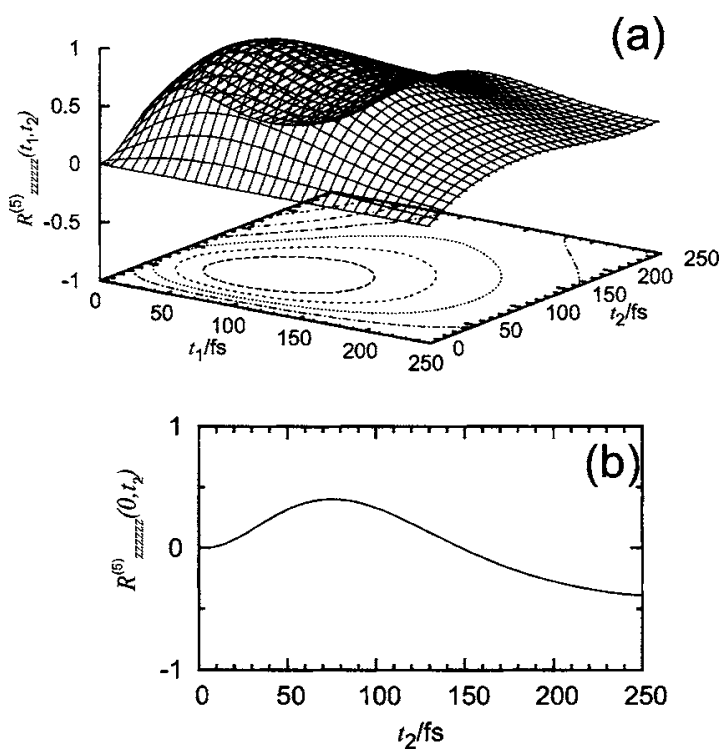

FIG. 6. (a) The $z z z z z z$ tensor element of the fifth-order response function of acetonitrile. The intensity of the signal is normalized by the peak intensity of the signal. (b) The slice of the $z z z z z z$ tensor element along the $t_{2}$ axis.

Both acetonitrile and $\mathrm{CS}_{2}$ have axial symmetric polarizabilities. In addition, acetonitrile has a large dipole moment parallel to the symmetry axis of the polarizabilities in contrast to $\mathrm{CS}_{2}$ which does not exhibit any dipole moment. The profiles of the fifth-order response functions of acetonitrile and $\mathrm{CS}_{2}$ are very similar, which indicate that either the effects of dipole-dipole interactions of acetonitrile molecules are not so important for the liquid dynamics at least at this temperature or 2D Raman is insensitive to the molecular motions caused by the dipole-dipole interactions.

The relative importance between the nonlinear polarizability (NL) and anharmonicity (AN) of vibrational motions can be detected from the 2D signals along the $t_{2}$ axis. In the case of $\mathrm{CS}_{2}$, it is shown that the AN exhibits as the negative part of the signal, while NL appears as the positive part. ${ }^{32}$ The 2D profile along the $t_{2}$ axis is depicted in Figs. 2(b), 3(b), 4(b), 5(b), and 6(b). The signals for $\mathrm{CS}_{2}$ [Fig. 3(b)] and acetonitrile [Fig. 6(b)] have positive area for small $t_{2}$, while those of water [Fig. 4(b)] and formamide [Fig. 5(b)] always exhibit negative signals. This indicates that the contribution of $\mathrm{AN}$ is larger than that of $\mathrm{NL}$ for the case of $\mathrm{CS}_{2}$ and acetonitrile. The analysis along the $t_{2}$ axis is simple, but is not suitable to see a role of the stability matrix which reflects the strength of dynamical coupling between the different modes, since the stability matrix element disappears along the $t_{2}$ axis due to the definition of its form. We also have

TABLE I. Relative intensities of the signals. The signal intensities are normalized by the peak signal intensity of $\mathrm{CS}_{2} . V$ is the volume of the system.

\begin{tabular}{cll}
\hline \hline Molecule & $\left|R^{(5)} / V\right|$ & $\left|R^{(3)} / V\right|$ \\
\hline $\mathrm{CS}_{2}$ & 1 & 1 \\
$\mathrm{Xe}$ & $2.1 \times 10^{-4}$ & $6.8 \times 10^{-3}$ \\
Water & $9.3 \times 10^{-3}$ & $6.4 \times 10^{-2}$ \\
Acetonitrile & $1.3 \times 10^{-2}$ & $7.5 \times 10^{-2}$ \\
Formamide & $1.9 \times 10^{-1}$ & $7.4 \times 10^{-1}$ \\
\hline
\end{tabular}

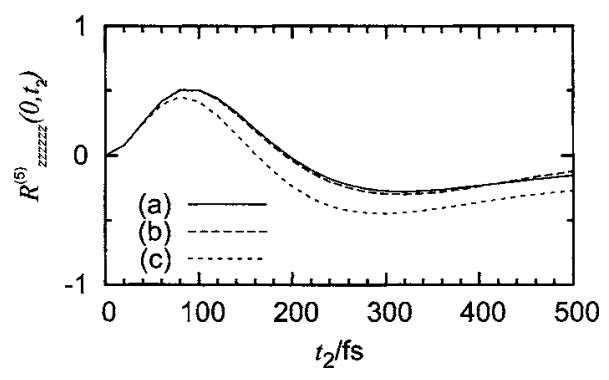

FIG. 7. The $z z z z z z$ tensor element of the fifth-order response function of $\mathrm{CS}_{2}$ along $t_{2}$ axis for different pulse strengths (a) $E=5 \mathrm{~V} / \AA$, (b) $E$ $=0.5 \mathrm{~V} / \AA$, and (c) $E=5 \mathrm{~V} / \AA$. (a) and (b) are calculated by the inverted force method. (c) is calculated without the inverted force method. In each figure, the signal intensity is normalized by the peak intensity of the signal. The cases (a) and (c) are averaged over for 2000000 initial configurations while the case (b) is averaged over for 4500000 initial configurations.

analyzed the signals along the $t_{1}=t_{2}$ (echo) direction and found that the signals for water and formamide have negative area, while those for the other liquids are always positive. This means that the contribution of AN from the stability matrix of water and formamide is stronger than that of other liquids, in other words, water and formamide have stronger collective motions. This is probably due to the hydrogen bonds formed in water and formamide liquids. Further studies of 2D signals for different temperatures and pressures in different phases including the analysis of the stability matrix elements $^{48,49}$ are necessary to have more detailed information of liquid dynamics.

\section{CONCLUSIONS}

We have developed the equilibrium and nonequilibrium hybrid MD simulation methods for the fifth-order response functions of 2D Raman spectroscopy. Since the response function in this method is expressed as the single Poisson bracket of the nonequilibrium trajectories with a single external perturbation, one can calculate the signal without evaluating the stability matrix elements. In addition, since we do not have to perform another NEMD calculation when changing $t_{2}$, this method enables us to calculate the fifthorder response function much faster than the conventional nonequilibrium method.

The convergence of the response function by using this method depends on the strength of the laser pulses; the stronger the laser pulses, the better convergence it is observed. The contaminations by higher-order signals, however, are observed when strong laser pulses are used. The hybrid method with the inverted force method which removes contaminations is more suitable for calculating the response functions even though it requires more calculations than the method without the inverted forces does. The fifth-order response functions of $\mathrm{Xe}$ and $\mathrm{CS}_{2}$ are in good agreement with those calculated previously by the equilibrium time correlation function or NEMD method, ${ }^{30,33}$ which means that the hybrid method is reliable. The hybrid method is very attractive to calculate the fifth-order response functions because this method requires much less computational cost than the others do, particularly for the large size systems. The relative intensities of the peak signals have been calculated and the 
obtained intensities of the fifth-order response function are related to the anisotropy of the polarizability. The relative contribution between the nonlinear polarizability and anharmonicity of vibrational modes is investigated for various molecules liquids. The results for formamide and water indicate that 2D Raman spectroscopy is a good probe to study the roles of the hydrogen bonds.

\section{ACKNOWLEDGMENTS}

The authors would like to thank S. Saito and Y. Nagata for very helpful discussions. One of the authors (Y.T.) is thankful for the financial support from a Grant-in-Aid for Scientific Research A15205005 from the Japan Society for the Promotion of Science and Morino Science Foundation.

\section{APPENDIX: CONVERGENCE OF THE FIFTH-ORDER RAMAN SIGNAL FOR DIFFERENT LASER STRENGTHS}

In this Appendix, we illustrate the convergence of the fifth-order response function upon our simulations as the function of the pulse strength for a case of $32 \mathrm{CS}_{2}$ molecules. Figure 7 depicts the 2D-Raman signal of $\mathrm{CS}_{2}$ for a various laser strengths. To obtain the reliable results, the signals are averaged over $2 \times 10^{6}$ initial configurations for (a) and (c) $E=5 \mathrm{~V} / \AA$, while $4.5 \times 10^{6}$ initial configurations for (b) $E$ $=0.5 \mathrm{~V} / \AA$. The signals (a) and (b) are almost the same, which indicates that the signals are almost independent of the field strengths in these calculations. Here we employ the inverted force method to eliminate a higher-order contribution of optical processes involved in the nonequilibrium part of our simulations. Figure 7(c) shows the 2D-Raman signals calculated without the inverted force method. In this case, the signal (c) shows strong deviations from the true fifthorder signals shown as (a) and (b). This is due to the contaminations of signal by the higher-order optical processes involved in our hybrid formalism. When strong laser pulses are used, the higherorder contributions play a dominant role if we do not employ the inverted forces method. Though the inverted force method requires one more NEMD calculation, we can save the CPU time, since the calculations with strong laser pulses by inverted force method require fewer number of initial configuration samplings.

${ }^{1}$ S. Mukamel, Principles of Nonlinear Optical Spectroscopy (Oxford University Press, Oxford, 1995).

${ }^{2}$ Y. Tanimura and S. Mukamel, J. Chem. Phys. 99, 9496 (1993).

${ }^{3}$ K. J. Kubarych, C. L. Milne, S. Lin, V. Astinov, and R. J. D. Miller, J. Chem. Phys. 116, 2016 (2002).

${ }^{4}$ K. J. Kubarych, C. L. Milne, and R. J. D. Miller, Chem. Phys. Lett. 369, 635 (2003).

${ }^{5}$ L. J. Kaufman, J. Heo, L. D. Ziegler, and G. R. Fleming, Phys. Rev. Lett. 88, 207402 (2002).

${ }^{6}$ P. Hamm, M. Lim, and R. M. Hochstrasser, J. Phys. Chem. B 102, 6123 (1998)

${ }^{7}$ M. T. Zanni, S. Gnanakaran, J. Stenger, and R. M. Hochstrasser, J. Phys. Chem. B 105, 6520 (2001)

${ }^{8}$ S. Woutersen and P. Hamm, J. Phys. Chem. B 104, 11316 (2000).

${ }^{9}$ M. Khalil, N. Demirdöven, and A. Tokmakoff, J. Phys. Chem. A 107, 5258 (2003)

${ }^{10}$ K. Ohta, H. Maekawa, S. Saito, and K. Tominaga, J. Phys. Chem. A 107,
5643 (2003).

${ }^{11}$ J. B. Asbury, T. Steinel, C. Stromberg, S. A. Corcelli, C. P. Lawrence, J. L. Skinner, and M. D. Fayer, J. Phys. Chem. A 108, 1107 (2004).

${ }^{12}$ K. Okumura and Y. Tanimura, J. Chem. Phys. 106, 1687 (1997).

${ }^{13}$ K. Okumura and Y. Tanimura, J. Chem. Phys. 107, 2267 (1997).

${ }^{14}$ K. Okumura and Y. Tanimura, Chem. Phys. Lett. 277, 159 (1997).

${ }^{15}$ M. Cho, K. Okumura, and Y. Tanimura, J. Chem. Phys. 108, 1326 (1998); A. Piryatinski, V. Chernyak, and S. Mukamel, Chem. Phys. 266, 311 (2001); V. Khidekel, V. Chernyak, and S. Mukamel, J. Chem. Phys. 105, 8543 (1996); S. Hahn, K. Park, and M. Cho, ibid. 111, 4121 (1999); K. Park, M. Cho, S. Hahn, and D. Kim, ibid. 111, 4131 (1999); M. Cho, ibid. 111, 4140 (1999).

${ }^{16}$ Y. Tanimura, Chem. Phys. 233, 217 (1998).

${ }^{17}$ K. Okumura and Y. Tanimura, Chem. Phys. Lett. 278, 175 (1997).

${ }^{18}$ A. Tokmakoff, M. J. Lang, D. S. Larsen, G. R. Fleming, V. Chernyak, and S. Mukamel, Phys. Rev. Lett. 79, 2702 (1997).

${ }^{19}$ K. Okumura, A. Tokmakoff, and Y. Tanimura, J. Chem. Phys. 111, 492 (1999).

${ }^{20}$ K. Okumura, D. M. Jonas, and Y. Tanimura, Chem. Phys. 266, 237 (2001).

${ }^{21}$ K. Hyeon-Deuk and Y. Tanimura, J. Chem. Phys. 123, 224310 (2005).

${ }^{22}$ T. Steffen and Y. Tanimura, J. Phys. Soc. Jpn. 69, 3135 (2000).

${ }^{23}$ Y. Tanimura and T. Steffen, J. Phys. Soc. Jpn. 69, 4095 (2000)

${ }^{24}$ T. Kato and Y. Tanimura, J. Chem. Phys. 117, 6221 (2002).

${ }^{25}$ T. Kato and Y. Tanimura, J. Chem. Phys. 120, 260 (2004).

${ }^{26}$ A. Ishizaki and Y. Tanimura, J. Chem. Phys. 123, 014503 (2005).

${ }^{27}$ Y. Tanimura, J. Phys. Soc. Jpn. 75, 082001 (2006).

${ }^{28}$ J. T. Fourkas, Adv. Chem. Phys. 117, 235 (2001).

${ }^{29}$ T. I. C. Jansen, J. G. Snijders, and K. Duppen, J. Chem. Phys. 113, 307 (2000)

${ }^{30}$ T. I. C. Jansen, J. G. Snijders, and K. Duppen, J. Chem. Phys. 114, 10910 (2001).

${ }^{31}$ S. Saito and I. Ohmine, Phys. Rev. Lett. 88, 207401 (2002)

${ }^{32}$ S. Saito and I. Ohmine, J. Chem. Phys. 119, 9073 (2003).

${ }^{33}$ A. Ma and R. M. Stratt, Phys. Rev. Lett. 85, 1004 (2000).

${ }^{34}$ R. A. Denny and D. R. Reichman, J. Chem. Phys. 116, 1979 (2002).

${ }^{35}$ J. Cao, J. Wu, and S. Yang, J. Chem. Phys. 116, 3739 (2002).

${ }^{36}$ J. Cao, J. Wu, and S. Yang, J. Chem. Phys. 116, 3760 (2002)

${ }^{37}$ T. Steffen, J. T. Fourkas, and K. Duppen, J. Chem. Phys. 105, 7364 (1996).

${ }^{38}$ T. Steffen and K. Duppen, Chem. Phys. 233, 267 (1998).

${ }^{39}$ M. Kryskov and J. Cao, J. Chem. Phys. 122, 024109 (2005).

${ }^{40}$ M. Kryvohuz and J. Cao, Phys. Rev. Lett. 95, 180405 (2005).

${ }^{41}$ T. Keyes and J. T. Fourkas, J. Chem. Phys. 112, 287 (2000).

${ }^{42}$ J. Kim and T. Keyes, Phys. Rev. E 65, 061102 (2002).

${ }^{43}$ T. Keyes and J. Kim, J. Chem. Phys. 122, 244502 (2005).

${ }^{44}$ R. DeVane, C. Ridley, B. Space, and T. Keys, J. Chem. Phys. 123, 194507 (2005).

${ }^{45}$ A. Ma and R. M. Stratt, J. Chem. Phys. 116, 4962 (2002).

${ }^{46}$ A. Ma and R. M. Stratt, J. Chem. Phys. 116, 4972 (2002).

${ }^{47}$ T. I. C. Jansen, K. Duppen, and J. G. Snijders, Phys. Rev. B 67, 134206 (2003).

${ }^{48}$ Y. Nagata and Y. Tanimura, J. Chem. Phys. 124, 024508 (2006).

${ }^{49}$ Y. Nagata, T. Hasegawa, and Y. Tanimura, J. Chem. Phys. 124, 194504 (2006)

${ }^{50}$ C. Dellago and S. Mukamel, J. Chem. Phys. 119, 9344 (2003).

${ }^{51}$ B. M. Ladanyi, Chem. Phys. Lett. 121, 351 (1985).

${ }^{52}$ A. Dullweber, B. Leimkuhler, and R. McLachlan, J. Chem. Phys. 107, 5840 (1997)

${ }^{53}$ M. P. Bogaard, A. D. Buckingham, R. K. Pierens, and A. H. White, J. Chem. Soc., Faraday Trans. 1 74, 3008 (1978).

${ }^{54}$ P. Moore and T. Keyes, J. Chem. Phys. 100, 6709 (1994)

${ }^{55}$ W. L. Jorgensen, J. Chandrasekhar, J. D. Madura, R. W. Impey, and M. L. Klein, J. Chem. Phys. 79, 926 (1983).

${ }^{56}$ C. Huiszoon, Mol. Phys. 58, 865 (1986).

${ }^{57}$ K. P. Sagarik and R. Ahlrichs, J. Chem. Phys. 86, 5117 (1987).

${ }^{58}$ M. C. Wojcik, K. Hermansson, and H. O. G. Siegbahn, J. Chem. Phys. 113, $3374(2000)$.

${ }^{59}$ J. Applequist, J. R. Carl, and K.-K. Fung, J. Am. Chem. Soc. 94, 2952 (1972).

${ }^{60}$ J. Richardi, P. H. Fries, and H. Krienke, J. Chem. Phys. 108, 4079 (1998) 